(RESEARCH ARTICLE)

\title{
A study of anatomical variation of external jugular vein
}

\author{
Suresh R Rao ${ }^{1,2,{ }^{*}}$ and T Ramesh Rao ${ }^{3}$ \\ ${ }^{1}$ Department of Anatomy, Subbaiah Institute of Medical Sciences, Shivamogga, India. \\ 2 Department of Anatomy Srinivasa Institute of Medical Sciences Mukka, Mangalore, India. \\ ${ }^{3}$ Department of Preclinical Sciences, Faculty of Medical Sciences, The University of the West Indies, St. Augustine Trinidad \\ and Tobago.
}

Publication history: Received on 08 July 2020; revised on 21 July 2020; accepted on 22 July 2020

Article DOI: https://doi.org/10.30574/wjarr.2020.7.1.0252

\begin{abstract}
The prior anatomical knowledge of variations of superficial veins of head and neck is important for head and neck surgeons, radiologists doing catheterization and to clinicians in general. Color Doppler Sonography is crucial for sensing collateral pathways. In cases of variations of the superficial veins of the neck, ultrasound guided venipuncture is potential procedure which may be achieved. The variations of the superficial veins of head and neck though common are important clinically. The superficial veins, especially the external jugular vein (EJV), are increasingly being utilized for cannulation to conduct diagnostic procedures or intravenous therapies. EJV is also used in microsurgical procedures, used as a recipient for the free flaps. The study therefore was intended at unfolding variant anatomy of external jugular vein as seen in a sample of Trinidadian population. Fifty-two sides of the neck from 26 cadaveric specimens (19 males and 7 females) in the anatomy unit, The University of West Indies, Trinidad were used. The level of formation, course, communications and termination were explored by dissection. In $41 \%$ of cases the vein was formed within the substance of the parotid gland, it was duplicated in $2 \%$ of cases and terminated into the internal jugular vein in $5 \%$ of cases. Awareness of these variations is important for the surgeons and clinicians performing head and neck vascular or reconstructive surgeries to avoid any intraoperative error procedures which might lead to unnecessary complications.
\end{abstract}

Keywords: External Jugular vein; Variations; Duplication

\section{Introduction}

External Jugular vein is usually formed by the confluence of the posterior auricular vein with the posterior division of retromandibular vein near the angle of the mandible just below or within the substance of parotid gland. It runs obliquely, caudally and dorsally, across the sternocleidomastoid muscle to a point opposite the middle of the clavicle, where it terminates in the corresponding subclavian vein. It is covered by platysma muscle and few branches of transverse cervical nerve. The great auricular nerve accompanies it in the superior half of its course. It lies on the superficial layer of cervical fascia which invests the sternocleidomastoid muscle. Just superior to the clavicle it perforates the superficial layer of investing layer of deep cervical fascia, by which it is prevented from collapsing, the fascia being attached to its walls. It then opens into the subclavian vein (occasionally into the internal jugular, or into the confluence of the subclavian and internal jugular veins). The external jugular vein may be absent or small, in which case the anterior jugular vein and or the internal jugular vein will be enlarged. Tributaries and communications of external jugular vein include the posterior auricular, posterior division of retromandibular, occipital, posterior external jugular, transverse cervical, suprascapular and anterior jugular veins [1]. Review of literature demonstrates that the variations in the patterns of its course, termination [2,3], duplication of EJV [4], fenestration [5], Phlebectasia [6], saccular aneurysm [7], anastomosis between the EJV and internal jugular vein [8,9]. Aforementioned knowledge of the existence of these variations plays important role in avoiding diagnostic pitfalls or therapeutic adversities. This study is directed at unfolding the variations of the external jugular vein in Trinidadian population.

\footnotetext{
* Corresponding author: Suresh R Rao
} 


\section{Material and methods}

Dissections of main blood vessels of the head and neck with a purpose of updating the data about anatomical variations of the EJV and preparation of specimens for teaching and museum were performed in the Gross Anatomy Laboratory on a total of fifty-two sides of the neck from 26 cadaveric specimens ( 19 males and 7 females) routinely used by year one \& two medical students for dissection in the anatomy unit were dissected to study the anatomical variation of the EJV. There were no any signs of trauma, surgery or wound scars of the neck in all cases. Systematically the skin flaps of the head and neck were reflected laterally. The superficial fascia of the neck, platysma was removed bilaterally from its proximal attachment and the EJV was exposed. The EJV was dissected and cleaned clearly to show its formation, course, tributaries and termination and was photographed.

\section{Results}

Fifty-two sides of the neck from twenty-six cadavers were dissected for the study of EJV. The vein was present in all the cases and it was formed by the union of posterior auricular vein and with the posterior division of retromandibular vein. In $41 \%$ of cases the EJV was formed within the substance of the parotid gland [Fig 1]. The EJV was duplicated in $2 \%$ of cases both are on the left side of the body in different individuals [Fig 2]. In both cases the vein was duplicated and rejoined again and terminated into the subclavian vein. In $5 \%$ of cases the EJV terminated at the junction of internal jugular vein and the subclavian vein [Fig.3].

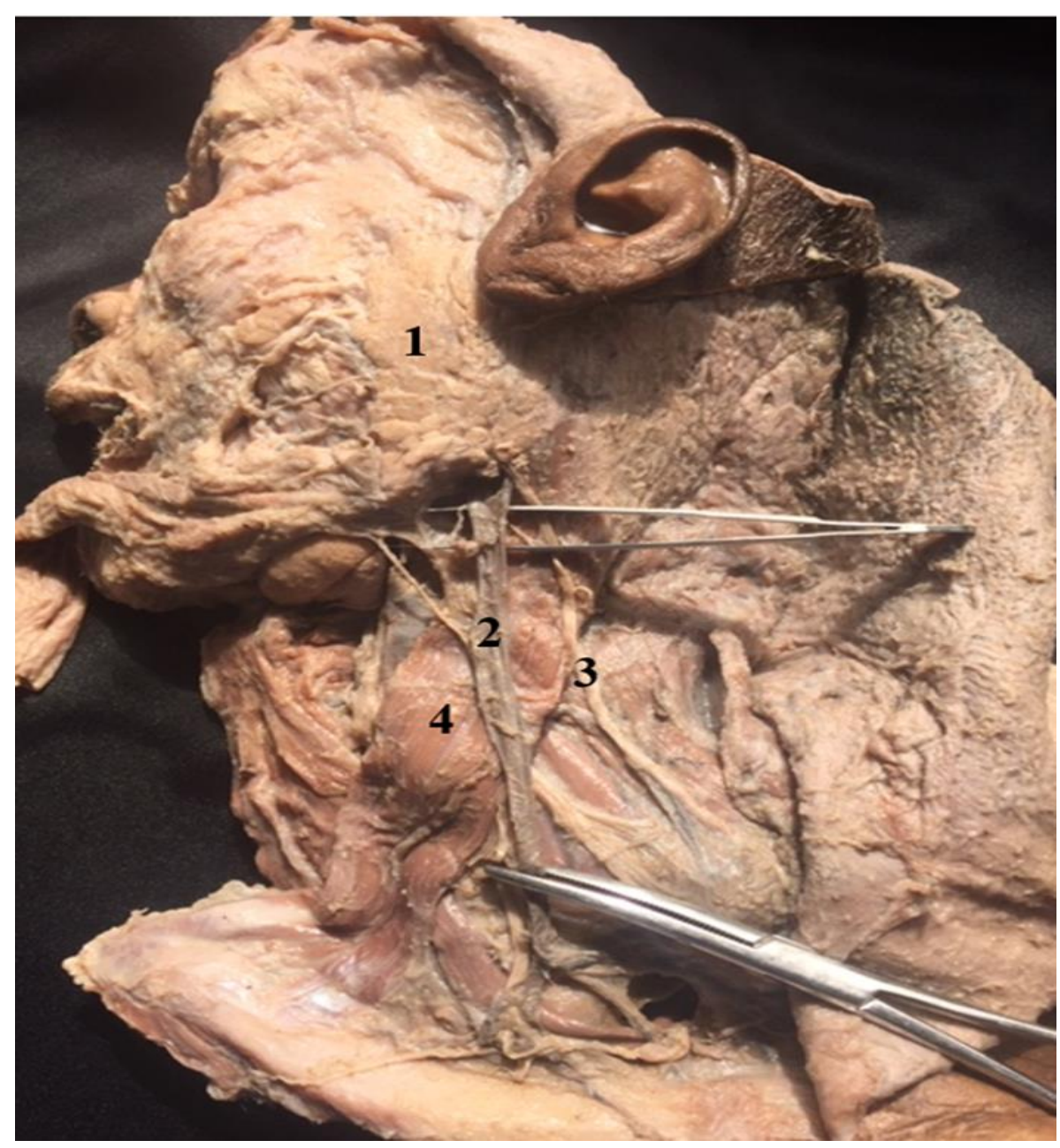

Figure 11. Parotid gland 2. External Jugular vein 3. Great auricular nerve 4. Sternocleidomastoid 


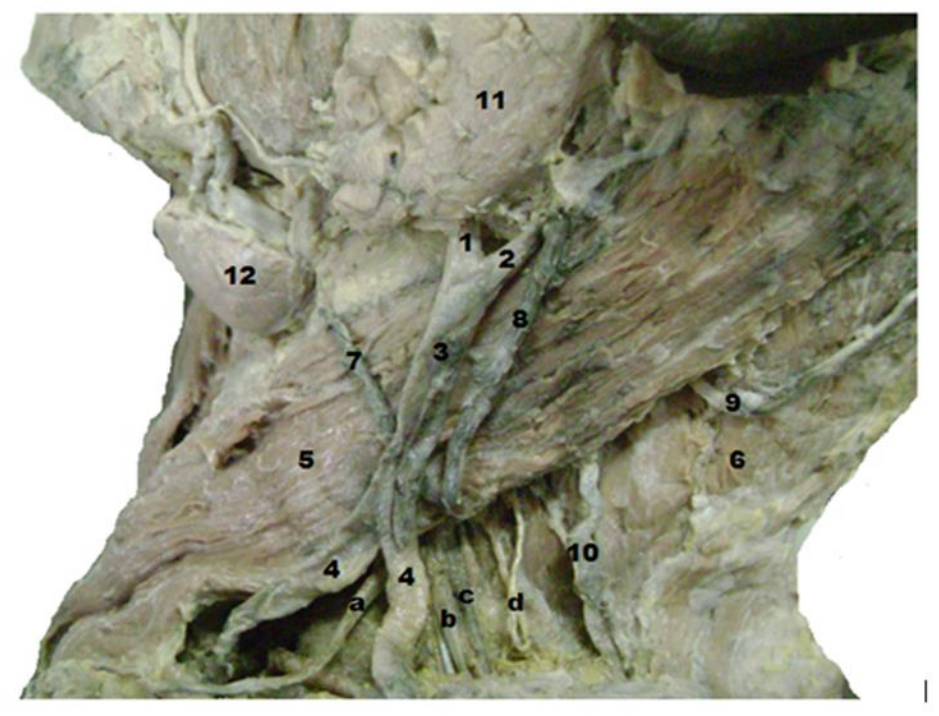

Figure 2 1. Posterior division of retromandibular vein 2. Posterior auricular vein 3. External jugular vein 4 . Two divisions of external jugular vein 5. Sternocleidomastoid muscle 6. Trapezius muscle 7. Transverse cervical nerve 8. Great auricular nerve 9. Lesser occipital nerve 10. Spinal accessory nerve 11. Parotid gland 12. Sub-mandibular gl and a-Medial branch of supra-clavicular nerve b. Intermediate branch of supra-clavicular nerve c. Lateral branch of supraclavicular nerve d. C3, C4 fibres

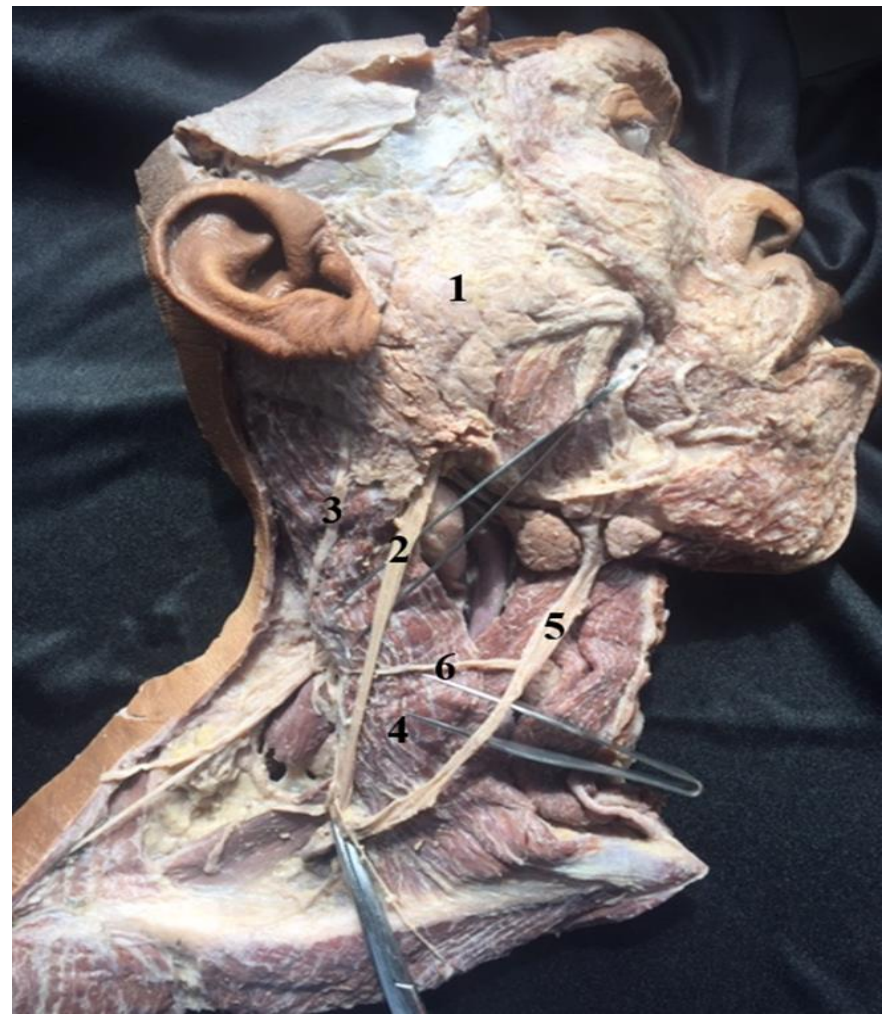

Figure 3 1. Parotid gland 2. External Jugular vein 3. Great auricular nerve 4. Sternocleidomastoid 5. Common facial vein 6 . Transverse cervical nerve

\section{Discussion}

Among other vascular systems the venous system represents a special interest for medical professional involved in diagnosis and management of vascular diseases. Deviations of embryonic development of blood vessels from the most common patterns are frequently encountered and widely recognized. A complicated process of transformation of the 
embryonic head vein, which involves regression and disappearance, retention, or reappearance of its components, may result in congenital anatomical variations in the origin and courses of the vessels [10].

Variations in the formation, course, communications and termination reported in the review of literature are so variable that it is not possible to determine a "normal" pattern [11]. Both EJV veins may be very small, or larger than the other, it may be absent on one or both sides; it may terminate into the internal jugular, cephalic or subclavian vein. It may be formed purely by the posterior auricular vein. On incident it receives the facial, lingual and cephalic veins. The vein is at times duplicated. The external jugular vein may descend posterior to the inferior belly of the omohyoid $[12,13]$. The present case also describes that the $41 \%$ of formation of EJV within the substance of the parotid gland. Since the EJV showing enormous variations, this should be kept in mind when attempting for any surgical procedures on the vein.

In the present study the It was found that the duplication of EJV in $2 \%$ of the cases. Comert E and Comert A [4], report observations on a patient operated on for head and neck cancer in which they observed duplication of the external jugular vein. The external jugular vein was seen duplicated in the middle third near the posterior border of sternocleidomastoid muscle before penetrating deep fascia. The external jugular vein is a preferred option in the head and neck free tissue transfer as the recipient vein and for central venous catheterization, hence this variation alerts clinicians and surgeons performing neck, vascular, or reconstructive surgery about unexpected variations of the external jugular vein in the hope of preventing inadvertent injury.

For the most part, the termination of the EJV into the ipsilateral subclavian vein is explained in all standard anatomy text books. In the present study $5 \%$ of the cases we found that the EJV is draining at the junction of internal jugular vein and subclavian vein. Kim and Han 2010 [14] also reported on the same pattern of termination of EJV. Several reports on the termination of EJV into the internal jugular vein has been documented [15-17]. Awareness of these variations is important for the surgeons to avoid any intraoperative trial or error procedures which might lead to unnecessary bleeding.

Superficial veins of head and neck develop from superficial plexus of the capillaries, which will ultimately form primary head vein. Larger channels are formed by enlargement of individual capillaries, confluence of adjacent ones, and regression of some from where the flow has been diverted. The factors controlling the selection and differentiation of the appropriate channels are not yet completely understood [18].

The superficial veins, especially the external jugular vein, are increasingly being utilized for cannulation to conduct diagnostic procedures or intravenous therapies. Ultrasound-guided venipuncture is a viable possibility in cases of variations in the patterns of superficial veins, and their knowledge is also important for surgeons doing reconstructive surgery. These veins are usually grafted into the carotid during endarterectomy and also used for surgery involving microvascular anastomosis especially in oral reconstruction procedures [19]

\section{Conclusion}

Information about details and topographic anatomy of the reported and other variations of EJV may serve as a useful guide for both radiologists and vascular surgeons. It can help to prevent diagnostic errors, influence surgical and interventional procedures and avoid surgical complications during head and neck surgery. Variations of EJV may be asymptomatic, so an extra care must be undertaken even during routine surgical interventions.

\section{Compliance with ethical standards}

\section{Acknowledgments}

We sincerely thank UWI and Subbaiah Institute of Medical Sciences for providing the opportunity to conduct this research.

\section{Disclosure of conflict of interest}

The author(s) declare that they have no competing interests. 


\section{References}

[1] Anson BJ. (1966). Morris Human Anatomy. 12th ed., New York, Mc Graw-Hill Book Company, 795-796.

[2] Choudhry R, Tuli A and Choudhry S. (1997). Facial vein terminating in the external jugular vein. An Embryologic interpretation. Surg Radiol Anat, 19(2), 73-77.

[3] Bergman RA, Afifi AK and Miyauchi R. (2011). Illustrated encyclopedia of human anatomic variation: Opus II: Cardiovascular system: Veins, Hed, Neck, and thorax.

[4] Comert E and Ciomert A. (2009). External jugular vein duplication. J Craniofac Surg , 20(6), 2173-2174.

[5] Cvetko E. (2012). A case of unilateral fenestration of the external jugular vein, through which the cervical branch of the facial nerve passes. Anat. Sci. Int., Epub ahead of print.

[6] Hermans R. (1991). Phlebectasia of the external jugular vein: a case report. J Belge Radiol, 74(3), 221-2.

[7] Verbeeck N, Hammer F, Goffette P and Mathurin P. (1997). Saccular aneurysm of the external jugular vein, an unusual cause of neck swelling. J Belge Radiol, 80(2), 63-4.

[8] Chalian AA, Anderson TD, Weinstein GS and Weber RS. (2001). Internal jugular vein versus external jugular vein anastomosis: Implications for successful free tissue transfer. Head Neck, 23(6), 475-8.

[9] Lalwani R, Rana KK, Das S and Khan RQ. (2006). Communication of the external and internal jugular veins: a case report. Int.J. Morphol, 24(4), 721-2.

[10] Moore KL. (1988). The developing human: Clinically oriented embryology 4th edition. Philadelphia: W.B Saunders, 291, 314.

[11] Bergman RA, Thomson SA, Afifi AK and Saadeh FA. (1988). Compendium of Human Anatomic Variation. Baltimore, Urban \& Schwarzenberg, 89.

[12] Cunnigham DJ. The vascular System; Abnormalities of Veins, 1058.

[13] Stickle BR and McFarlane H. (1997). Prediction of a small internal jugular vein by external jugular vein diameter. Anaesthesia, 52, 220-222.

[14] Kim DI and Han SH. (2010). Venous variations in the Neck Region: Cephalic vein. International Journal of Anatomical Variations, 3, 208-210.

[15] Standring S. (2005). Gray's anatomy. 39th ed., London, Elsevier Churchil Livingstone, 445.

[16] Hollinshead WH. (1956). Anatomy for surgeons. IInd Ed. Hoeber-Harper, New York, V.I., 530-1.

[17] Shailaja CM, K Sandyha and GB Rairam. (2006). Varoatopm om the ter,omatopm pf exterma; kigi;ar veom -A case report: Journal of the anatomical Society of India, 55(1).

[18] Yadav S, Ghosh SK and Anand C. (2000). Variations of Superficial Veins of Head and Neck Journal of Anatomical Society of India, 49(1), 61-62.

[19] Chauhan AK, Rani A, Chopra J, Rani A, Srivastava AK and Kumar V. (2011). Anomalous formation of external jugular vein and its clinical implication. Natl J Maxillofac Surg, 2(1), 51-53.

\section{How to cite this article}

Suresh RR and T Ramesh R. (2020). A study of anatomical variation of external jugular vein. World Journal of Advanced Research and Reviews, 7(1), 197-201. 